\title{
Analysis of Fuzzy Non-preemptive Priority Queuing Model with Unequal Service Rate
}

\section{K. Selvakumari ${ }^{a^{*}}$ and S. Revathi ${ }^{\mathrm{b}}$}

${ }^{a *}$ Professor, Department of Mathematics, Vels Institute of Science, Technology and Advanced Studies, Chennai, Tamilnadu, India. E-mail: selvafeb6@gmail.com

${ }^{\mathrm{b}}$ Research Scholar, Department of Mathematics, Vels Institute of Science, Technology and Advanced Studies, Chennai, Tamilnadu, India. E-mail: revathiraja1867@gmail.com

Article History: Received: 11 January 2021; Accepted: 27 February 2021; Published online: 5 April 2021

\begin{abstract}
This article provides an effective method to analyze the performance measures of non-preemptive fuzzy priority queues with unequal service rates. Here the arrival rate and the service rate are in fuzzy numbers. Using a new ranking method, the fuzzy values are reduced to the crisp values. For that cause, both the Triangular Fuzzy Number (TFN) and Trapezoidal Fuzzy Number (TpFN) are chosen to establish the proposal's effectiveness. An illustration is given to find the efficiency of the performance measures of the fuzzy queuing model.
\end{abstract}

Keywords: Fuzzy Queuing Model, Triangular Fuzzy Number (TFN), Trapezoidal Fuzzy Number (TpFN).

\section{Introduction}

Nowadays, the concept of Queuing theory has pervasive applications in the real-time hypothesis. Overall, the Priority Queues has a wide range of applications like communication networks, transport sector, healthcare management, etc. At the same time, the concept of Fuzzy Queues is also extensively debated. Li and Lee (1989), Negi and Lee (1992), Kao and Wilson (1999), Chen (2005, 2006) are some of the researchers who developed the concept of Fuzzy Queues. Section 3 proposes a new ranking method in this paper, and section 4 intends to apply the proposal effectively.

\section{Preliminaries}

\subsection{Fuzzy Set}

A Fuzzy set $\widetilde{F}$ is defined by $\widetilde{F}=\left\{\left(x, \mu_{\widetilde{F}}(x)\right): x \in X, \mu_{\widetilde{F}}(x) \in[0,1]\right\}$. In the pair of an ordered set $\left(x, \mu_{\widetilde{F}}(x)\right)$, the first element $x$ belongs to the Universe $X$, and the second element $\mu_{\widetilde{F}}(x)$ belongs to the interval $[0,1]$, then the set $\widetilde{\mathrm{F}}$ is called a fuzzy set and $\mu_{\widetilde{F}}(\mathrm{x})$ is called the Membership function.

\subsection{Crisp Set}

For any crisp set $\widetilde{\mathrm{F}}$, it is defined as the characteristic function $\mu_{\widetilde{\mathrm{F}}} \rightarrow\{0,1\}$, i.e., the characteristic function takes either of the value 0 or 1 in the classical set.

\subsection{Triangular Fuzzy Number}

The Triangular Fuzzy Number $(\mathrm{TFN})$ represented three points: $\widetilde{F}=\left(\widetilde{f}_{1}, \widetilde{f}_{2}, \widetilde{f}_{3}\right)$. This representation is interpreted as membership functions and satisfies the following conditions

$$
\mu_{\widetilde{\mathrm{F}}}(\mathrm{x})=\left\{\begin{array}{cc}
0 & \text { for } \mathrm{x}<f_{1} \\
\frac{\left(\mathrm{x}-f_{1}\right)}{f_{2}-f_{1}} & \text { for } f_{1} \leq \mathrm{x} \leq f_{2} \\
\frac{\left(f_{3}-\mathrm{x}\right)}{f_{3}-f_{2}} & \text { for } f_{2} \leq \mathrm{x} \leq f_{3} \\
0 & \text { for } \mathrm{x}>f_{3}
\end{array}\right.
$$

(i) $\widetilde{f}_{1}$ to $\widetilde{f}_{2}$ is a monotonically increasing continuous real-valued function.

(ii) $\widetilde{f}_{2}$ to $\widetilde{f}_{3}$ is monotonically decreasing continuous real-valued function

(iii) $\widetilde{f}_{1} \leq \widetilde{f}_{2} \leq \widetilde{f}_{3}$.

\subsection{Trapezoidal Fuzzy Number}


The Trapezoidal Fuzzy Number $(\mathrm{TpFN})$ is represented with three points: $\widetilde{F}=\left(\widetilde{f_{1}}, \widetilde{f}_{2}, \widetilde{f_{3}}, \widetilde{f_{4}}\right)$. This representation is interpreted as membership functions and satisfies the following conditions.

$$
\mu \widetilde{\mathrm{F}}(\mathrm{x})=\left\{\begin{array}{cc}
0 & \text { for } \mathrm{x}<f_{1} \\
\frac{\left(\mathrm{x}-f_{1}\right)}{f_{2}-f_{1}} & \text { for } f_{1} \leq \mathrm{x} \leq f_{2} \\
1 & \text { for } f_{2} \leq \mathrm{x} \leq f_{3} \\
\frac{\left(f_{3}-\mathrm{x}\right)}{f_{3}-f_{4}} & \text { for } f_{3} \leq \mathrm{x} \leq f_{4} \\
0 & \text { for } \mathrm{x}>f_{4}
\end{array}\right.
$$

(i) $f_{1}$ and $f_{2}$ is real-valued monotonically increasing continuous real-valued function

(ii) $f_{3}$ and $f_{4}$ is real-valued monotonically decreasing continuous real-valued function

(iii) $f_{1}<f_{2} \leq f_{3}<f_{4}$

\subsection{Models and Description}

\subsubsection{Non-pre preemptive priority with unequal service rates}

Let us consider a single server two-class non-preemptive priority queue with different service rates. The interarrival rate of $\lambda_{1}$ and $\lambda_{2}$ are distributed independently. The service rates $\mu_{1}$ and $\mu_{2}$ are also distributed independently. FCFS queue discipline is followed, whereas the low priority customer gets prior service than the high priority customers. From the classical queueing theory,

where,

$$
\begin{gathered}
L_{q}^{(1)}=\frac{\lambda_{1}\left[\frac{\lambda_{1}}{\mu_{1}{ }^{2}}+\frac{\lambda_{2}}{\mu_{2}{ }^{2}}\right]}{\left(1-\rho_{1}\right)} \\
L_{q}{ }^{(2)}=\frac{\lambda_{2}\left[\frac{\rho_{1}}{\mu_{1}}+\frac{\rho_{2}}{\mu_{2}}\right]}{\left(1-\rho_{1}\right)(1-\rho)}
\end{gathered}
$$

$$
\begin{aligned}
& \rho=\rho_{1}+\rho_{2} \\
& \rho_{1}=\frac{\lambda_{1}}{\mu_{1}}, \\
& \rho_{2}=\frac{\lambda_{2}}{\mu_{2}} \\
& \rho=\rho_{1}+\rho_{2}<1 \text { is mandatory for its steady-state. }
\end{aligned}
$$

\section{New Proposed Ranking Method}

To change the fuzzy values into real crisp values, we use the following new proposed ranking method.

$$
R(\tilde{F})=\frac{\left(\tilde{f}_{\min }+\tilde{f}_{\max }\right)}{2}
$$

whereas $\tilde{f}_{\min }$ and $\tilde{f}_{\max }$ are the minimum and maximum values of the given fuzzy number.

\section{Numerical Illustration}

Let us assume a critical situation happens in Prabhu Medical Clinic in Chennai, where some outpatients have arrived in need of medical treatment as a matter of urgency. In case of emergency, the doctor allows patients immediately to receive his attention and treatment (non-preemptive priority only). We now calculate the average queue length of that two-class non-preemptive priority outpatients queue on this contingency.

\subsection{Triangular Fuzzy Number}

Let $\lambda_{1}=[3,5,7]$ and $\lambda_{2}=[4,6,8]$ are the arrival rate and $\mu_{1}=[21,23,25]$ and $\mu_{2}=[22,24,26]$ are two different service rates respectively.

$$
R\left(\lambda_{1}\right)=\frac{7+3}{2}=5
$$




$$
\begin{aligned}
& R\left(\lambda_{2}\right)=\frac{4+8}{2}=6 \\
& R\left(\mu_{1}\right)=\frac{21+25}{2}=23 \\
& R\left(\mu_{2}\right)=\frac{22+26}{2}=24 \\
& \rho_{1}=\frac{\lambda_{1}}{\mu_{1}}=0.2174 \\
& \rho_{2}=\frac{\lambda_{2}}{\mu_{2}}=0.2500 \\
& \rho=\rho_{1}+\rho_{2}=0.4674
\end{aligned}
$$

\subsubsection{Results}

\begin{tabular}{ll}
\hline No. of. Priority & Average Queue Length $\left(\mathbf{L}_{\mathbf{q}}\right)$ \\
\hline First Priority & 0.1268 \\
Second Priority & 0.2861 \\
\hline
\end{tabular}

\subsection{Trapezoidal Fuzzy Number}

Let $\lambda_{1}=[11,13,15,17]$ and $\lambda_{2}=[12,14,16,18]$ are the arrival rate and $\mu_{1}=[27,29,31,33]$ and $\mu_{2}=$ $[28,30,32,34]$ are two different service rates respectively.

$$
\begin{aligned}
& R\left(\lambda_{1}\right)=\frac{11+17}{2}=14 \\
& R\left(\lambda_{2}\right)=\frac{12+18}{2}=15 \\
& R\left(\mu_{1}\right)=\frac{27+33}{2}=30 \\
& R\left(\mu_{2}\right)=\frac{28+34}{2}=31 \\
& \rho_{1}=\frac{\lambda_{1}}{\mu_{1}}=0.4667 \\
& \rho_{2}=\frac{\lambda_{2}}{\mu_{2}}=0.4839 \\
& \rho=\rho_{1}+\rho_{2}=0.9506
\end{aligned}
$$

\subsubsection{Results}

\begin{tabular}{ll}
\hline No. of. Priority & Average Queue Length $\left(\mathbf{L}_{\mathbf{q}}\right)$ \\
\hline First Priority & 0.81826 \\
Second Priority & 17.7438 \\
\hline
\end{tabular}

\section{Conclusion}

This paper examines the average queue length of the two-class non-preemptive priority queue with unequal service rate. The crisp values of the fuzzy arrival rate and the fuzzy service rate were calculated by a new ranking method. It is more efficient than other existing ranking method. It can be further applied for queuing models in the future.

\section{References}

1. Kalpana, B., \& Anusheela, N. (2018). Analysis of a Single Server Non-Preemptive Fuzzy Priority Queue using LR Method. ARPN Journal of Engineering and Applied Sciences, 13(23), 9306-9310.

2. Ramli, N., \& Mohamad, D. (2009). A comparative analysis of centroid methods in ranking fuzzy numbers. European Journal of Scientific Research, 28(3), 492-501.

3. Ritha, W., \& Robert, L. (2010). Fuzzy queues with priority discipline. Applied Mathematical Sciences, 4(12), 575-582.

4. Ramesh, R., \& Ghuru, S.K. (2014). Priority disciplined queuing models with fuzzy. Journal of Engineering and Applied Sciences, 4(3), 594-602. 
Analysis of Fuzzy Non-preemptive Priority Queuing Model with Unequal Service Rate

5. Kalpana, B., \& Anusheela, N. (2019). Analysis of Fuzzy Priority Queue with two classes of customers and unequal service rates using Mixed Integer Non-Linear Programming Approach. Journal Engineering and Applied Sciences 14(15), 5030-5038. 